\title{
Ca II Triplet Spectroscopy of Small Magellanic Cloud Red Giants. IV. Abundances for a Large Sample of Field Stars and Comparison with the Cluster Sample
}

\author{
M.C. Parisi \\ Observatorio Astronómico, Universidad Nacional de Córdoba \\ Laprida 854, Córdoba, CP 5000, Argentina. \\ Consejo Nacional de Investigaciones Científicas y Técnicas \\ Av. Rivadavia 1917, Buenos Aires, CP 1033, Argentina \\ celeste@oac.uncor.edu \\ D. Geisler \\ Departamento de Astronomía, Universidad de Concepción \\ Casilla 160-C, Concepción, Chile. \\ dgeisler@astro-udec.cl \\ G. Carraro \\ European Southern Observatory \\ Casilla 19001, Santiago de Chile, Chile. \\ gcarraro@eso.org \\ J.J. Clariá \\ Observatorio Astronómico, Universidad Nacional de Córdoba \\ Laprida 854, Córdoba, CP 5000, Argentina. \\ Consejo Nacional de Investigaciones Científicas y Técnicas \\ Av. Rivadavia 1917, Buenos Aires, CP 1033, Argentina \\ claria@oac.uncor.edu
}


S. Villanova

Departamento de Astronomía, Universidad de Concepción

Casilla 160-C, Concepción, Chile.

svillanova@astro-udec.cl

L.V. Gramajo

Observatorio Astronómico, Universidad Nacional de Córdoba

Laprida 854, Córdoba, CP 5000, Argentina.

luciana@oac.uncor.edu

A. Sarajedini

Department of Astronomy, University of Florida

PO Box 112055, Gainesville, FL 32611, USA.

ata@astro.ufl.edu

and

A.J. Grocholski

Department of Physics and Astronomy, Louisiana State University

202 Nicholson Hall, Tower Drive, Baton Rouge, LA 70803-4001, USA.

grocholski@phys.lsu.edu

Received

accepted 


\begin{abstract}
This paper represents a major step forward in the systematic and homogeneous study of Small Magellanic Cloud (SMC) star clusters and field stars carried out by applying the Calcium Triplet technique. We present in this work the radial velocity and metallicity of approximately 400 red giant stars in 15 SMC fields, with typical errors of about $7 \mathrm{~km} \mathrm{~s}^{-1}$ and 0.16 dex respectively. We added to this information our previously determined metallicity values for 29 clusters and approximately 350 field stars using the identical techniques. Using this enlarged sample, we analyze the metallicity distribution and gradient in this galaxy. We also compare the chemical properties of the clusters and of their surrounding fields. We find a number of surprising results. While the clusters, taken as a whole, show no strong evidence for a metallicity gradient, the field stars exhibit a clear negative gradient in the inner region of the SMC consistent with the recent results of Dobbie et al. (2014b). For distances to the center of the galaxy less than 4 degrees, field stars show a considerably smaller metallicity dispersion than that of the clusters. However, in the external SMC regions, clusters and field stars exhibit similar metallicity dispersions. Moreover, in the inner region of the SMC, clusters appear to be concentrated in two groups: one more metal-poor and another more metal-rich than field stars. Individually considered, neither cluster group presents a metallicity gradient. Most surprisingly, the metallicity gradient for both stellar populations (clusters and field stars) appears to reverse sign in the outer regions of the SMC. The difference between the cluster metallicity and the mean metallicity of the surrounding field stars turns out to be a strong function of the cluster metallicity. These results could be indicating different chemical evolution histories for these two SMC stellar populations. They could also indicate variations in the chemical behavior of the SMC in its internal
\end{abstract}


and external regions.

Subject headings: galaxies: stellar content — Magellanic Clouds — stars:abundances 


\section{Introduction}

The Small Magellanic Cloud (SMC) possesses a number of unique characteristics which make it a fundamental benchmark for a wide variety of astrophysical studies. Being the closest dwarf irregular galaxy, it is the ideal local analog for the detailed study of these most common and primeval galaxies. With its low metallicity, the SMC is an excellent laboratory for the exploration of the role of this key parameter. It is close enough to allow detailed studies of its stellar populations even in the oldest stars, allowing the possibility of deriving the intimate and global details about such key galactic parameters as its chemical evolution, entire star formation history, etc. Several fundamental diagnostics in this regard are the age-metallicity relation, metallicity distribution and metallicity gradient. The populous system of SMC star clusters has been successfully used in recent years for these purposes, as they provide very accurate points in space and time. Field stars can be used in addition to clusters when age information is not critical or all that is required are good abundances for a large sample of objects (e.g. Carrera et al. 2008; Cignoni et al. 2013; Dias et al. 2014; Dobbie et al. 2014b; Rubele et al. 2015; Piatti 2015, among others). However, despite its unique advantages, the SMC has been surprisingly underexploited and even a basic knowledge of many of the above parameters is scant, controversial or completely lacking.

Our group has been investigating abundances as well as velocities in SMC clusters and surrounding field stars using the powerful CaII (CaT) spectroscopy technique for a number of years (Parisi et al. 2009, 2015, hereafter P09 and P15, respectively). As shown by Cole et al. (2004) (hereafter C04), CaT is a very efficient and accurate metallicity indicator, with minimal age effects. We have previously obtained CaT spectra of more than 200 likely members in 29 populous SMC clusters spanning a very wide range of ages and metallicities with the VLT+FORS2. This is the most comprehensive and homogeneous spectroscopic dataset to date for studying the chemical evolution history of the SMC (P09 and P15). 
With the data thus obtained, we derived radial velocities (RVs) to a few $\mathrm{km} \mathrm{s}^{-1}$ per star and mean cluster metallicities to 0.05 dex. In addition, the preimages used to obtain the astrometry were purposely lengthened in order to go as deep as possible, and combined with the excellent seeing provided photometry better than anything so far available for virtually all of our clusters. We analyzed the color-magnitude diagramas (CMDs) built from these preimages in order to derive ages for all clusters and we found that three of them are indeed very old clusters previously unrecognized as such (Parisi et al. 2014). At the same time, we obtained similar data for a total of some 750 SMC field giants surrounding the clusters, which allows us to investigate RVs and metallicities for them but not their ages. The initial sample of 360 field stars were analyzed and presented in Parisi et al. (2010), (hereafter P10). With this high quality sample, we analyzed the metallicity distribution (MD), age-metallicity relationship (AMR) and metallicity gradient (MG) for the SMC. Also, we compared the chemical properties of clusters and surrounding field stars.

Among the many interesting results found in the above cited works, one of the most curious and controversial is the nature of any MG. The existence of a MG in the SMC has been the subject of substantial debate for years (e.g. Piatti et al. 2007a,b; Carrera et al. 2008; Cioni 2009; P09; P10). The recent work by Dobbie et al. (2014b) (hereafter D14b) provides strong new evidence concerning field stars. They found, from CaT metallicity of several thousand field stars, a clear negative MG in the inner region of the galaxy. However, the system of star clusters studied by us in P15, with the same technique, does not show convincingly any gradient, perhaps suggesting that clusters and field stars have not undergone the same history, or that clusters have been more spatially mixed than field stars. Also in P15 we found evidence for a possible V-shape in the ([Fe/H],semi-major axis) diagram, with a vertex around $4-5^{\circ}$, just beyond the outer limit of the D14b data, and we suggested the possibility of an inversion of the MG in the outer region of the SMC. This strange behavior, if real, is very difficult to explain. However, the sample of points is 
small, especially in the critical outer regions where the deviant behavior appears. These are precisely the regions in which the kinematic study of Dobbie et al. (2014a) has shown that the tidal interaction between the SMC and LMC, and between the Magellanic Clouds and the Milky Way, are probably more evident.

A second intriguing result involves the MD. Our previous field star sample (P10) found a mean of $[\mathrm{Fe} / \mathrm{H}]=-1.00 \pm 0.02$ with no evidence for multi-modality. However, our full cluster sample (P15) yielded a high probability that the MD is bimodal, with peaks at -1.1 and -0.8 dex. To add to this controversy, D14b also find unimodality in their field star sample, with a median metallicity of $-0.99 \pm 0.01$, virtually identical to P10, while Mucciarelli (2014) observed some 200 SMC field giants with the high resolution VLT+FLAMES/GIRAFFE spectrograph and derived MD peaks of about -1.0 with a secondary peak at -0.6 . Clearly, these major mysteries in one of our closest galactic neighbors require further careful investigation to try and arrive at the definitive understanding.

As a natural continuation of our previous work, in this paper we derive the metallicity of some 400 red giant stars from 15 SMC fields with the main objective to continue investigating the chemical properties of this galaxy, especially the MD and MG. This provides a field star sample more than double that we analyzed previously (P10) and yields a large, high quality, homogenous dataset with which to compare with our cluster results obtained simultaneously. In Section 2 we describe the target selection and the observations. In Section 3 we explain the RV, equivalent width and metallicity determinations. In Section 4 we specify the final field sample and Section 5 is devoted to the metallicity analysis. Finally, we summarize our results in Section 6. 


\section{Target selection and observations}

This paper is a continuation of our previous work wherein we determined and analized the metallicity of 29 SMC clusters (P09 and P15) and that of a sample of $\sim 350$ red giant field stars (P10) surrounding the 15 SMC star clusters studied in P09. Here we add the $\sim 400$ red giants in 15 fields that surround the 14 star clusters studied in P15 (in P15 we discard the cluster B113, but we can still use the surrounding field stars). We repeat here the identical procedures followed in P10. We refer the reader to P15 for details regarding the selection of the cluster sample, the spectroscopic targets and the observations. The list of the star clusters studied in P15 can be seen in Table 1 of that paper. Figure 1 shows the distribution of the current fields studied as well as those studied in P10. For reasons related to the analysis made in Section 5, we have divided Figure 1 into eight sectors, marking the fields belonging to each of them with a different colour.

Each cluster was centered on the master chip while surrounding field stars were observed in both the master and secondary CCD. Pre-images in the $V$ and $I$ bands and the spectra of selected stars were obtained as part of programs 0.82B-0505 and 384.B-0687. The selection of spectroscopic targets was made on the corresponding $(V, V-I)$ CMD by choosing stars located along the red giant branch. The spectroscopic observations were performed in service mode with the instrument FORS2 on the VLT in mask exchange unit mode (MXU), with the same instrumental setup as in Grocholski et al. (2006) and P09. A very few stars have $\mathrm{S} / \mathrm{N} \sim 15$ pixel $^{-1}$, but the remaining targets have $\mathrm{S} / \mathrm{N}$ ratio between $\sim 20$ and $\sim 80$ pixel $^{-1}$. Information about exposure times, size of the slits, seeing and characteristics of the obtained spectra can be found in Section 3 of P15. Moreover, Grocholski et al. (2006) and P09 include a full description of the reduction and analysis techniques applied in the present work. We emphasize that all datasets (two each for both clusters and their surrounding field stars) were obtained with identical observational setups 
and reduced in an identical manner.

\section{Equivalent width measurements and metallicity determinations}

In Section 4 of P15 we describe the measurement of RVs and equivalent widths (EWs) of the observed red giants. In Section 5 of the same work, we detail the procedure followed for target metallicity determination. Although this paper as well as those of C04; Grocholski et al. (2006); P09 and P10 contain all the information concerning our measurement and analysis procedure, we include here a brief summary of these aspects for the sake of completeness.

The RV values of the field stars observed in this paper, as well as the values of those studied in P10, will be published in a forthcoming paper where the kinematics of the SMC field stars will be analyzed.

The program of C04 was used to measure the EW of the three CaT lines, adopting the rest-frame wavelengths and continuum bandpasses from Armandroff \& Zinn (1988) and fitting a Gaussian or a Gaussian + Lorentzian function to each CaT line, according to the criteria explained in detail in P15. The $\Sigma W$ index represents the sum of the EWs of the three CaT lines.

We removed the effects of surface gravity and temperature as well as the dependence on distance and interstellar reddening on $\Sigma W$ by a calibration using the difference in $\mathrm{V}$ magnitude between the red giant in question and the level of the red clump, through equation (4) of $\mathrm{C} 04$. The value of $\beta$ derived by these authors was used in order to maintain homogeneity with our previous work. The difference in (uncalibrated) $V$ magnitude between the corresponding star and that of the red giant clump $\left(V_{H B}\right)$ of the cluster was measured from aperture photometry on the pre-images. Equation (5) of C04 was finally used to 
derive the individual metallicity of each observed star, with errors ranging from 0.09 to 0.32 dex and a mean of 0.16 dex. In Table 1, we list the measured values for each individual field star. Column 1 shows the identification of the star, while the following columns give in succession the measured $v-v_{H B}, \sigma_{\Sigma W}$ and its error and the metallicity $([\mathrm{Fe} / \mathrm{H}])$ and its error.

\section{Field sample}

In P09 and P15 we performed a membership analysis to discriminate stars belonging to the clusters from their surrounding field stars. The membership method applied involves a combination of four different criteria: position along the main red giant branch, distance from the cluster center, RV, and metallicity of the observed red giants. The details of this procedure can be seen in P15. The field star sample analyzed in the current work includes the stars observed in the secondary CCD, plus those observed in the main chip which did not fulfill all of the above criteria and, therefore, are not considered cluster members. For stars taken from the master CCD, we verified that their metallicities and RVs were not compatible with those of the cluster values. Any potential cluster contamination on the secondary chip should be negligible considering that this chip is fairly well separated from

the master one. We discard from the samples those red giants having RVs outside the range accepted for SMC members $\left(50<\mathrm{RV}<250 \mathrm{~km} \mathrm{~s}^{-1}\right.$, Harris \& Zaritsky 2006). For the subsequent analysis, we also added to our present sample ( $\sim 400$ red giants) the field stars studied in P10. This makes for a combined sample of $\sim 750$ SMC field stars, one of the largest available. 


\section{Metallicity analysis}

\subsection{Metallicity distribution}

The MD of our full sample of field stars (this work + P10) is shown in the left panel of Figure 2. If we fit a Gaussian function to this sample, we find an $[\mathrm{Fe} / \mathrm{H}]$ value for the peak of $-0.97 \pm 0.01$, with a dispersion of $0.30 \pm 0.01$. However, using a field sample much larger than ours (3037 stars), D14b recently showed that the MD departs from a Gaussian shape showing a tail towards the metal-poor region. Note that there is a suggestion of this excess metal-poor tail in our MD below $[\mathrm{Fe} / \mathrm{H}] \sim-1.5$ as well, although it is clearly less evident than in D14b. This is most likely due to the lower number of stars dealt with in this paper. However, there is no indication of any further deviation from a simple Gaussian shape in our MD, such as bimodality. The mean $[\mathrm{Fe} / \mathrm{H}]$ value derived from our sample is $-1.00 \pm 0.01$, while the value corresponding to the "median metallicity" coincides with the peak of the fitted Gaussian function, i.e., $[\mathrm{Fe} / \mathrm{H}]=-0.97 \pm 0.01$. All of these values show excellent agreement with D14b's median metallicity $(-0.99 \pm 0.02)$. Although we used a Gaussian function and not a theoretical metallicity distribution function to fit the MD, we inferred that the intrinsic abundance dispersion implied from the observed abundance one is around 0.3 dex. This value was obtained taking into account a statistical dispersion error of 0.09 dex.

We combined our field sample with the one from D14b in the right panel of Figure 2. The fit of a Gaussian function to the combined sample gives a metallicity peak of $-0.976 \pm$ 0.007, in excellent agreement with values mentioned in the previous paragraph. Mucciarelli (2014) very recently obtained high resolution VLT+FLAMES/GIRAFFE spectra for a sample of 214 SMC field giants, deriving detailed abundances for a variety of elements including Fe. These stars belong to the three fields surrounding clusters NGC 419, NGC 339 and NGC 121, covering a spatial area smaller than that of our study. His $[\mathrm{Fe} / \mathrm{H}]$ 
distribution shows a primary peak very similar to that found above, around -0.9/-1.0. However, he also finds a secondary peak at $[\mathrm{Fe} / \mathrm{H}] \sim-0.6$. The relative sizes of the primary and secondary peaks are $\sim 3 / 1$. There is no evidence in either our data or that of D14b for this more metal-rich peak. Possible explanations include small number statistics in the case of the Mucciarelli (2014) study, some hitherto unknown effect between the CaT and high resolution metallicities, which seems very difficult to be the case as it requires a non-linear effect to explain the excess in one vs. the other technique, or some other reason.

To further complicate matters, we remind the reader that in our analysis of the full cluster sample, P15 found a clear signature of bimodality with a probability of $96 \%$, with peaks at about $[\mathrm{Fe} / \mathrm{H}]=-1.1$ and -0.8 . We compare in Figure 3 our field star and cluster MDs. Surprisingly, the field peak falls clearly in the dip between the two cluster peaks. We compare the two stellar populations (field stars and star clusters) by applying Kolmogorov-Smirnov statistics to investigate whether star clusters and surrounding fields come from the same parent population. There is a very low probability $(\mathrm{P}=0.04)$ of this occuring, which stresses the significant difference between the samples. When considering only metal-poor clusters and fields $([\mathrm{Fe} / \mathrm{H}] \leq-1)$, such probability increases, becoming as high as $\mathrm{P}=0.86$ in the $[\mathrm{Fe} / \mathrm{H}] \leq-1$ metallicity range.

Despite the above apparent significant differences in the cluster and field star MDs, our derived mean cluster metallicity of $-0.9 \pm 0.2$ dex shows very good agreement with the above mean field star value. It is at least clear that the mean metallicity of SMC giants in both the field and in clusters to which the CaT technique is applicable (generally for stars older than about $1 \mathrm{Gyr}$ ) is very close to -1 . However, it is essential to obtain good metallicities for an even larger sample of both clusters and field stars to explore the reality of these intriguing possible differences and the true nature of the field and cluster MDs. 


\subsection{Cluster vs. surrounding field star metallicities}

How does the mean metallicity of each individual cluster compare to its surrounding field? While the orbits of both populations, clusters and field stars, may be different and therefore their chemical evolution may be unrelated, it is still of interest to compare them, as we did in P10.

In order to perform a robust comparative metallicity analysis, we need to first calculate a representative metallicity for each field. The criterion used in P10 was to fit a Gaussian function to the MD of each field, with a few exceptions due to small number statistics (L7, L17 and L106). We adopted the peak of the Gaussian as the representative metallicity of each field. However, D14b suggested that the median metallicity of each field is more appropriate than the Gaussian peak due to the potential departure of the MD from a Gaussian shape. For the present sample, we calculate both values in order to compare with both P10 as well as D14b. We list in Table 2 the name of the selected field in column (1), the number of stars $n$ belonging to the corresponding field in column (2), the peak of the fitted Gaussian function $\left([\mathrm{Fe} / \mathrm{H}]_{G}\right)$ in columns (3) and the median metallicity value $\left([\mathrm{Fe} / \mathrm{H}]_{M}\right)$ and the standard error of the median are shown in column (4). Figures $4-7$ show the Gaussian fits for the 15 selected fields studied in the present work.

In Table 3 we present $[\mathrm{Fe} / \mathrm{H}]_{M}$ and its corresponding error for the fields studied in $\mathrm{P} 10$, while the $[\mathrm{Fe} / \mathrm{H}]_{G}$ values can be seen in Table 3 of P10. As can be seen in Tables 2 and 3 there is good agreement between $[\mathrm{Fe} / \mathrm{H}]_{G}$ and $[\mathrm{Fe} / \mathrm{H}]_{M}$ in the metallicity range covered by our current sample. The difference between $[\mathrm{Fe} / \mathrm{H}]_{M}$ and $[\mathrm{Fe} / \mathrm{H}]_{G}$ (in absolute value) ranges from 0.01 to 0.08 with a mean value of 0.02 and standard deviation of 0.02 . The difference, including sign, ranges from -0.07 to 0.08 , with a mean value and standard deviation of -0.003 and 0.032 , respectively. There is no clear indication that using the peak of a Gaussian function as the representative global value is significantly different or less appropriate than 
using the median value in the metallicity range here considered. However, we will use the median metallicities in the subsequent analysis, for the sake of consistency with the work of D14b. Also note that our sample has three fields (H86-97, B113 and HW47) in common with those studied by D14b (C3, C4 and 3D15F). The differences in mean metallicity are $0.02,0.08$ and 0.13 dex, respectively. The corresponding distributions have similar dispersions. Both studies are consistent with each other within the respective errors.

In P10 we concluded that most SMC fields (represented by their Gaussian peak) show a clear tendency to be more metal-poor than the corresponding cluster, regardless of their position in the galaxy or the cluster age. We also found that the difference between cluster and surrounding field metallicity exhibits a trend in the sense that the difference decreases with increasing cluster metallicity. With the aim of verifying these intriguing findings, we use our larger field and cluster (P15) star sample in order to plot the metallicity difference between these two stellar populations $\left([\mathrm{Fe} / \mathrm{H}]_{\text {field }}-[\mathrm{Fe} / \mathrm{H}]_{\text {cluster }}\right)$ as a function of the semi-major axis a (Figure 8), age (Figure 9) and cluster metallicity (Figure 10). Triangles and circles represent the original (P09, P10) and followup (P15, this paper) samples, respectively. To facilitate identification of the different points in the subsequent figures, we recommend the reader to keep in mind that all information on star clusters can be found in Table 3 of P09 and in Table 3 of P15. The relevant data concerning stellar fields are contained in Table 3 of P10 and in Tables 2 and 3 of the present work. As can be deduced from these three figures, the above mentioned tendencies are clearly present in our enlarged field and cluster star sample and thus their reality is now more robust. Figures 8 and 9 show that most of the field star representative metallicities are more metal-poor than their corresponding cluster mean, irrespective of the cluster age and position in the galaxy. However, there are several exceptions to this tendency represented by some of the most metal-poor clusters. 
Figure 10 clearly shows even more clearly the trend previously found in P10 in the sense that the difference between the metallicity of the two populations is strongly linearly correlated with $[\mathrm{Fe} / \mathrm{H}]_{\text {cluster }}$. We note that the fields surrounding the clusters more metal-rich than $[\mathrm{Fe} / \mathrm{H}]=-1$ are more metal-poor than their respective clusters (with the lone exception of L106), while for clusters more metal-poor than $[\mathrm{Fe} / \mathrm{H}]=-1$, the difference $[\mathrm{Fe} / \mathrm{H}]_{\text {field }}-[\mathrm{Fe} / \mathrm{H}]_{\text {cluster }}$ ranges from -0.15 to 0.33 dex, the mean value and standard deviations being $0.08 \pm 0.18$.

At least part of this difference can be understood in light of the different MDs of field and cluster stars. The field stars are unimodal with peak near -1 while the clusters appear bimodal, with peaks on either side of the field peak. Thus, to first order, the more metal-rich clusters will be more metal-rich than their surrounding field stars while the opposite holds for the more metal-poor clusters. In addition, there are more metal-rich than metal-poor clusters in our sample, causing the excess of fields which are metal-poorer than their clusters as opposed to metal-richer. However, the strong correlation must have further explanation. A possibility is that this partly stems from the field stars being somewhat older and therefore somewhat more metal-poor than most of our clusters, given the age-metallicity relation of the SMC (P15). The metallicity differences between these two populations could be hinting at a different chemical history for them or at a different spatial distribution, perhaps due to SMC line-of-sight depth effects, which is hard to investigate given our lack of information regarding the age and distance of our star field stars. An important factor lessening confidence in our results is the relatively small size of the total sample, which is susceptible to small number statistics. Again, efforts to increase the sample size will help to further stregthen the significance of this curious result. The metallicity values of the cluster sample studied by Kayser et al. were reported by Glatt et al. (2008b) but they have not yet been published. We are analyzing the ESO archive public data to perform our own determinations for this sample (Gramajo et al., in preparation). This will not only add a 
new cluster sample but also the stars belonging to their surrounding fields, which will be homogeneously analyzed following the same procedures as in the present work.

\subsection{Metallicity gradient}

The existence or not of a MG can be crucial for understanding the stellar formation and chemical evolution processes in a galaxy. Important efforts to confirm or deny the existence of a MG in the SMC have been made using photometry and spectroscopy for both field and cluster stars (e.g., Piatti et al. 2007a,b; Carrera et al. 2008; Cioni 2009; P09; P10; D14b; P15). Despite these concerted efforts, there is no consensus about the nature of any MG.

Piatti et al. (2007a,b) used Washington photometry of a cluster sample and found that the mean metallicity values and the respective metallicity dispersions tended to be higher for the clusters located within $4^{\circ}$ from the SMC center than for those situated outside this radius. However, from a Washington study of a large sample of field stars, Piatti (2012) claimed that field stars have not gradients in either age or metallicity, finding no difference in the AMR between clusters and field stars. Their cluster metallicities had typical error bars of about 0.2 dex, but also included a significant age correction for stars younger than 5 Gyr when using their standard giant branch technique. In addition, the field stars values are only qualitatively-weighted means for a huge number of stars per field.

Large-scale CaT spectroscopic determinations began with Carrera et al. (2008), who obtained metallicities of a sample of some 350 field red giants in 13 fields distributed from $\sim 1^{\circ}-4^{\circ}$ in radius from the center. They found a mean metallicity of $[\mathrm{Fe} / \mathrm{H}] \sim-1.0$ in the innermost SMC fields, with the mean decreasing in the outermost regions, reaching -1.6 at $4^{\circ}$ radius. However, almost all of the power in the MG they find lies in their two outermost 
fields, which have the smallest sample size and thus largest metallicity errors. They also found a relationship between this MG and their age gradient in the sense that the youngest stars, concentrated in the central regions, were the most metal-rich. It is interesting to note that Cioni (2009) find no MG from asymptotic giant branch stars.

In our initial CaT sample of 15 clusters (P09) and their surrounding fields (P10), no evidence for a MG in either of these separate or combined samples appears. However, when we added the additional clusters studied in P15, we found a suggestion of a MG in our full cluster sample, at least within the innermost $4^{\circ}-5^{\circ}$, although we could not confirm it quantitatively. Beyond that, any potential gradient becomes very flat or in fact turns around and becomes positive. We noted that from $0-2.5^{\circ}$, the MG appears to be flat. At larger distances, the mean metallicity decreases, exhibiting a minimum at $4-5^{\circ}$. Finally, the mean metallicity rises to become flat again in the outermost parts, which include several fields as far as $8^{\circ}$ away from the center. Quantitative assessment of any MG was hampered by the large intrinsic cluster metallicity dispersion at all radii.

Quite recently, D14b found a clear $-0.075 \pm 0.011 \mathrm{dex} / \mathrm{deg}$ gradient, based on CaT metallicities of about 3000 field giants. They found a tight correlation out to their outermost point at $4.5^{\circ}$ from the center. They interpreted the MG to be the result of an increasing fraction of young stars with decreasing galactocentric radius, coupled with a uniform global age - metallicity relation. Opposite to this result is the work of Deb et al. (2015). They do not detect any MG from the analysis of more than 1000 RR Lyrae stars.

As the number of SMC stars analyzed at high resolution is small and limited to only a few fields, no measurement of the MG based on this technique exists.

In order to assess the possible existence of a MG in the SMC as carefully as possible, we revisit this parameter, now adding these additional field stars to our previous CaT field and cluster star sample. This is the largest homogenous spectroscopic study involving 
both field and cluster stars available to date and should shed light on this intriguing issue. First, however, the orientation of the galaxy and projection effects must be addressed. The orientation is unfortunately poorly determined, although it is believed the galaxy is markedly elongated along the line of sight (Gardiner \& Hawkins 1991; Subramanian \& Subramanian 2012; Haschke et al. 2012; Jacyszyn-Dobrzeniecka et al. 2016), making projection effects important but the determination of true galactocentric distances difficult to ascertain. We have followed all of our previous work and utilized the procedure described by Piatti et al. (2007a), which adopts an elliptical coordinate system (Figure 1, Paper I) and computed for each field the value of the semimajor axis, $a$, which an ellipse would have under the following conditions: (1) if it were centered on the SMC center, (2) if it were aligned with the bar, (3) had a b/a ratio of $1 / 2$, and (4) if one point of the trajectory coincided with the field position. Then, we use the semi-major axis $a$ value as a surrogate for the true galactocentric distance. Most other studies cited above adopt this same procedure.

Figure 11 (left panel) shows the behaviour of the metallicity as a function of the semi-major axis $a$. Black symbols represent the clusters analyzed in P15: black filled circles are clusters studied in P09 and P15, while clusters from Da Costa \& Hatzidimitriou (1998) and Glatt et al. (2008a,b) are represented by open circles and triangles, respectively. Metallicities of clusters studied by Da Costa \& Hatzidimitriou (1998) and Glatt et al. (2008a,b) were also derived from CaT lines. Following P15, we include these objects, as they should be on a very similar metallicity scale to ours. Red symbols stand for our field stars: the sample studied here (circles) and the one from P10 (triangles). The right panel of 11 shows the same as the left panel, with clusters and fields separated in order to help the reader to better visualize the data for the two stellar populations.

As noted in our previous studies (P15), SMC clusters appear to have a considerable 
metallicity dispersion, which is significantly larger than the errors involved in the cluster metallicity calculations. A linear fit to our cluster data yields a MG which is null within the errors. However, field stars present a considerably smaller metallicity dispersion compared to the star clusters. Taking into account only field stars, a significant MG does indeed exist, at least within the first $4-5^{\circ}$ from the SMC center, as reported by D14b. A linear fit to our field data (solid line in Figure 11) yields a metallicity gradient of $-0.08 \pm 0.02 \mathrm{dex} \mathrm{deg}^{-1}$, in excellent agreement with that of $-0.075 \pm 0.011 \mathrm{dex} \mathrm{deg}^{-1}$ found by D14b. This study is totally independent of the work of D14b. As such, the results regarding the metallicity gradient are further strengthened.

Therefore, although we confirm the metallicity gradient derived by D14b for the SMC field stars, we emphasize, as we did in P15, that our cluster data do not show a clear unbroken variation of the metallicity as a function of semi-major axis $a$, taken as a whole. We stress again the fact that both our clusters and fields have metallicities determined on a homogeneous scale, from observations made with the same telescope and instrumental configuration, and by applying the same procedure. Therefore, cluster and field metallicities can be directly compared. But it is curious to see that, if we consider clusters at a distance less than $4^{\circ}$, they appear to be concentrated in two groups: one above the sequence of the fields and one below, which we call the metal-rich group and metal-poor group, respectively. These are the two populations which emerge in the MD. As can be seen in Figure 11, independent linear fits to each of these groups (dotted lines) show that neither group has

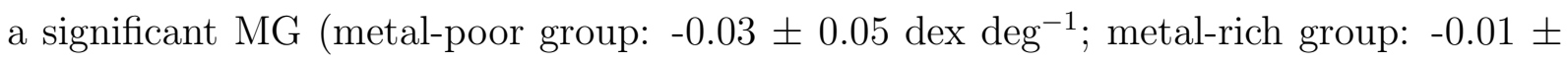
$\left.0.02 \mathrm{dex} \mathrm{deg}^{-1}\right)$. However, if we extend the boundary of the inner region from $4^{\circ}$ to $5^{\circ}$, including the two black points and the triangle at $[\mathrm{Fe} / \mathrm{H}] \sim-1$, we find values of $-0.06 \pm$ $0.02 \mathrm{dex} \mathrm{deg}^{-1}$ and $-0.04 \pm 0.03 \mathrm{dex} \mathrm{deg}^{-1}$, for the metal-rich and for the metal-poor group, respectively. The results are extremely dependent on the vertex we assume and on the edge definition made between the inner and the outer regions. The choice of the limit is of 
course arbitrary and there is no physical reason to prefer one to the other. The statistical significance of these two potential gradients is difficult to assess.

In Figure 12 is shown the distribution of metal-rich and metal-poor clusters with circles and triangles, respectively. Metal-rich clusters appear to be homogeneusly distributed, while the metal-poor clusters are mostly located in sectors 6 , with the exception of two clusters. In any case, the number of clusters considered is indeed too small. We can not conclude from this study that the metallicity difference between these two groups, if it exists, is a consequence of the spatial distribution. Dias et al. (2014) and Dias el al. (2016) divide clusters from the Bica et al. (2008)'s catalog into four groups: Main body, Wing/bridge, Bridge and West Counter-halo (see Figure 8 of Dias et al. 2014), but our sample is too small to analyze, homogeneously, the metallicity gradient in each of these structures.

The most remarkable phenomenon in Figure 11 is the clear inversion of the MG observed beyond $4-5^{\circ}$. We estimated the distribution of linear model slopes for random samples with distances less and greater than $4^{\circ}$, obtained from 500 bootstrap resamplings. The gradient inversion is significant at the $99 \%$ level $(87 \%$ if we consider a turning point at $5^{\circ}$ ). In P15 we called attention to the apparent V-shape of the (weak if any) cluster MG. This inversion is now much more graphically seen in the field stars, which do have a clear inner negative MG but also clearly reverse this trend in the outer regions. Both populations display this trend but the large intrinsic spread in the clusters at a given radius limits the ability to discern this bizarre behavior. It is indeed curious that the most metal-rich cluster HW86 is in one of the most distant regions studied, and the outermost field has a metallicity rivalling that of the innermost field, which is the most metal-rich of all the fields. It is important to note that Dobbie et al. (2014b) independently evaluated the metallicity of HW86 derived by Parisi et al. (2009), finding a very good agreement. So we believe that the metallicity of this cluster is well determined. 
Another curious phenomenon appears: of the 22 clusters in the inner region (again differentiating at $\left.5^{\circ}\right), 18$ are more metal-rich than their surrounding fields, while in the outermost 7 clusters, only 4 are more metal-rich, although here we are fighting small number statistics.

In an effort to elucidate possible causes for this inversion, in Figure 13 we show the MG only for field stars, now using the colour code introduced in Figure 1 for different azimuthal sectors. Six of the seven outer regions responsible for the reversal all lie in sectors 3 and 4 . This is in the direction towards the Magellanic Bridge. Since the clusters lie in the same positions as their surrounding fields, the same tendency holds for them. These clusters are not distinguished by their ages, covering the full range of the entire sample from 1.4 to 7.6 Gyr (P14). Six other fields lying in these sectors in the inner region fall amongst their peers from other sectors in metallicity.

The fact that the change in the slope of the MG occurs in the outer parts of the galaxy in the direction of the Magellanic bridge is certainly interesting. One is tempted to suggest that this phenomenon could then be due to the past interaction of the SMC with the LMC. Another aspect to consider is the depth of the SMC. Cole et al. (2004) and Koch et al. (2006) showed that the associated error in $[\mathrm{Fe} / \mathrm{H}]$, due to the use of an inappropriate $\mathrm{V}_{H B}$ value, is around 0.05 dex (as large as 0.1 dex in extreme cases). Even if these errors were taken into account to determine our metallicities, the behavior of the observed gradient would not change. But we do not rule out an effect due to the fact that we are using a projected reference system (the semi-major axis $a$ instead of the real distance). However, it should be noted that our knowledge of the details of the inversion, if indeed real, are at present very superficial. We are still far from having a definitive picture of the MG. The sample size is very small and possible selection effects may be at work, e.g. we have no clusters in sectors 7 and 8, i.e., in the opposite direction to the Magellanic bridge. 
The number of clusters in these outer regions is small in any case and one may be forced to observe only field stars. Clearly, this fascinating phenomenon demands independent verification using as large a sample as possible. Our group is attempting to achieve this goal.

\section{Summary}

From CaT lines we have determined the metallicity of $\sim 400$ field red giant stars belonging to the surrounding fields of 15 SMC clusters. We have added these stars to those studied in P10 compiling a final sample of $\sim 750$ field stars. We found a median metallicity for this sample of $-0.97 \pm 0.01$ in very good agreement with previous works. Metallicity distribution of field stars shows considerable differences with the corresponding metallicity distribution of clusters (P10, P15).

For each of the 30 fields (15 from this work + P10) we calculated the median metallicity and analyzed the behavior of metallicity as a function of the semi-major axis $a$. Also we compared the field and cluster (P09+P15) gradient. In the inner region of the galaxy

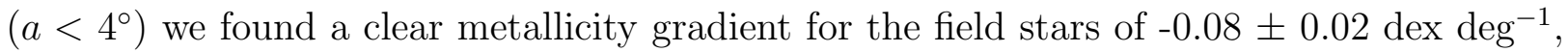
in excellent agreement with D14b, but a considerable difference between the metallicity dispersion of fields and clusters. On the other hand, in the outer part of the SMC $\left(a>4^{\circ}\right)$ the metallicity of clusters and field stars show very similar values (with the exception of one point, HW86) and both populations appear to suggest the existence of a positive external gradient. In order to confirm this suggestion, is it necessary to determine metallicity for a larger sample of cluster and field stars located beyond $4^{\circ}$ and especially in the opposite direction to the Bridge in order to properly test the suggestion that the inversion is an effect due to the interaction between the SMC and LMC or a selection effect. 
Finally we compared field metallicities (this work + P10) with the metallicities of the corresponding clusters (P09 + P15). We confirm our previous findings that most fields are more metal poor that the corresponding cluster, independently of the position in the galaxy and the cluster age. Also, as in P10 we found a linear dependence of the difference between cluster and field metallicity with cluster metallicity.

The current study is based on observations collected at the European Southern Observatory (Chile), under programs numbers 0.82.B-0505 and 384.B-0687. We would like to thank the Paranal Science Operations Staff. We would also like to thank Dr. Mucciarelli for his interesting discussion on the metallicity gradient. M.C.P., J.J.C. and L.V.G. gratefully acknowledge financial support from the Argentinian institutions CONICET, ANPCyT and SECYT (Universidad Nacional de Córdoba). D.G. gratefully acknowledges support from the Chilean BASAL Centro de Excelencia en Astrofísica y Tecnologías Afines (CATA) grant PFB-06/2007. S.V. gratefully acknowledges the support provided by FONDECYT reg. n. 1130721. We thank the referee for his/her detailed analysis of our work, which has contributed to the improvement of the present study.

Facilities: VLT: Antu (FORS2). 


\section{REFERENCES}

Armandroff, T.E., \& Zinn, R. 1988, AJ, 96, 92

Bica, E., Bonatto, C., Dutra, C. M., \& Santos, J. F. C. 2008, MNRAS, 389, 678

Carrera, R., Gallart, C., Aparicio, A., et al. 2008, AJ, 136, 1039

Cignoni, M., Cole, A.A., Tosi, M., Gallagher, J.S., Sabbi, E., Anderson, J., Grebel, E. K., Nota, A. 2013, ApJ, 775, 83

Cioni, M-R.L., 2009, A\&A, 506, 1137

Cole, A.A., Smecker-Hane, T.A., Tolstoy, E., Bosler, T.L., \& Gallagher, J.S. 2004, MNRAS, $347,367(\mathrm{C} 04)$

Da Costa, G.S., \& Hatzidimitriou, D. 1998, AJ, 115, 1934

Deb, S., Singh, H.P., Kumar, S., Kanbur, S.M. 2015, MNRAS, 449, 2768

Dias, B., Kerber, L.O., Barbuy, B., Bica, E., Ortolani, S. 2016, arXiv160403086D

Dias, B., Kerber, L.O., Barbuy, B., Santiago, B., Ortolani, S., Balbinot, E. 2014, A\&A, 561, 106

Dobbie, P.D., Cole, A.A., Subramaniam, A., Keller, S. 2014, MNRAS, 442, 1663

Dobbie, P.D., Cole, A.A., Subramaniam, A., Keller, S. 2014, MNRAS, 442, 1680 (D14b)

Gardiner, L.T. \& Hawkins, M.R.S., 1991, MNRAS, 251, 174

Glatt, K., et al. 2008a, AJ, 135, 1106

Glatt, K., Grebel, E.K., Sabbi, E. et al. 2008b, AJ, 136, 1703

Grocholski, A.J., Cole, A.A, Sarajedini, A., Geisler, D., \& Smith, V. 2006, AJ, 132, 1630 
Harris, J., \& Zaritsky, D. 2006, AJ, 131, 2514

Haschke, R., Grebel, E.K., Duffau, S., 2012, AJ, 144, 107

Jacyszyn-Dobrzeniecka, A.M., Skowron, D.M., Mróz, P., Skowron, J., Soszyński, I., Udalski, A. et al. 2016, arXiv160209141J

Kayser, A., Grebel, E.K., Harbeck, D.R., Cole, A.A., Koch, A., Gallagher, J.S., \& Da Costa, G.S. 2006, preprint (astro-ph/0607047)

Koch, A., Grebel, E.K., Wyse, R.F.G., Kleyna, J.T., Wilkinson, M.I., Harbeck, D.R., Gilmore, G.F., \& Evans, N.W. 2006, AJ, 131, 895

Mucciarelli, A. 2014, AN, 335, 79

Parisi, M.C., Geisler, D., Carraro, G., Clariá, J.J., Costa, E., Grocholski, A.J., Sarajedini, A., Leiton, R., Piatti, A.E. 2014 AJ, 147, 71

Parisi, M.C., Geisler, D., Clariá, J.J., Villanova, S., Marcionni, N., Sarajedini, A., Grocholski, A.J. 2015, AJ, 149, 154 (P15)

Parisi, M.C., Geisler, D., Grocholski, A.J., Clariá, J.J., Sarajedini, A. 2010, AJ, 139, 1168 $(\mathrm{P} 10)$

Parisi, M.C., Grocholski A.J., Geisler, D., Sarajedini, A., \& Clariá, J.J. 2009, AJ, 138, 517 (P09)

Piatti, A.E., 2012, MNRAS, 422, 1109

Piatti, A.E. 2015, 2015, MNRAS, 451, 3219

Piatti, A.E., Sarajedini, A., Geisler, D., Clark, D., \& Seguel, J. 2007a, MNRAS, 377, 300 
Piatti, A.E., Sarajedini, A., Geisler, D., Gallart, C., \& Wischnjewsky, M. 2007b, MNRAS, 381, L84

Rubele, S., Girardi, L., Kerber, L. et al. 2015, MNRAS, 449, 639

Subramanian S. \& Subramaniam A., 2012, ApJ, 744, 128

Tonry, J., \& Davis, M. 1979, AJ, 84, 1511 
Table 1. Measured values for field stars

\begin{tabular}{cccccc}
\hline \hline ID & $v-v_{H B}$ & $\Sigma W$ & $\sigma_{\Sigma W}$ & {$[\mathrm{Fe} / \mathrm{H}]$} & $\sigma_{[\mathrm{Fe} / \mathrm{H}]}$ \\
& $(\mathrm{mag})$ & $(\AA)$ & $(\AA)$ & $(\mathrm{dex})$ & $(\mathrm{dex})$ \\
\hline & & & & & \\
B99M 1 & -1.805 & 7.073 & 0.075 & -0.883 & 0.107 \\
B99M 2 & -0.734 & 6.465 & 0.080 & -0.820 & 0.107 \\
B99M 3 & -1.401 & 5.410 & 0.060 & -1.378 & 0.091 \\
B99M 4 & -1.536 & 6.547 & 0.147 & -1.002 & 0.112 \\
B99M 5 & -1.093 & 6.224 & 0.078 & -1.002 & 0.102 \\
\hline
\end{tabular}

Note. - Table 1 is published in the electronic edition in its entirety. 
Table 2. SMC field results

\begin{tabular}{lccc}
\hline \hline & & & \\
\multicolumn{1}{c}{ ID } & $\mathrm{n}$ & {$[\mathrm{Fe} / \mathrm{H}]_{G}$} & {$[\mathrm{Fe} / \mathrm{H}]_{M}$} \\
\hline & & & \\
B 99 & 36 & $-0.87 \pm 0.04$ & $-0.90 \pm 0.05$ \\
B 113 & 37 & $-0.99 \pm 0.04$ & $-1.01 \pm 0.04$ \\
H 86-97 & 20 & $-0.76 \pm 0.05$ & $-0.76 \pm 0.05$ \\
HW 40 & 22 & $-0.97 \pm 0.04$ & $-0.98 \pm 0.04$ \\
HW 67 & 27 & $-0.94 \pm 0.04$ & $-0.96 \pm 0.05$ \\
K 3 & 18 & $-1.17 \pm 0.06$ & $-1.14 \pm 0.07$ \\
K 6 & 27 & $-1.06 \pm 0.05$ & $-1.05 \pm 0.07$ \\
K 8 & 40 & $-0.91 \pm 0.04$ & $-0.91 \pm 0.05$ \\
K 9 & 32 & $-0.92 \pm 0.04$ & $-0.93 \pm 0.05$ \\
K 37 & 35 & $-0.96 \pm 0.04$ & $-0.96 \pm 0.05$ \\
K 44 & 26 & $-0.95 \pm 0.05$ & $-0.95 \pm 0.06$ \\
L 1 & 12 & $-1.25 \pm 0.08$ & $-1.19 \pm 0.10$ \\
L 112 & 19 & $-1.14 \pm 0.05$ & $-1.07 \pm 0.04$ \\
L 113 & 9 & $-0.96 \pm 0.03$ & $-0.93 \pm 0.05$ \\
OGLE 133 & 40 & $-0.82 \pm 0.04$ & $-0.84 \pm 0.04$ \\
\hline \hline
\end{tabular}


Table 3. Median metallicities of previously studied SMC fields

\begin{tabular}{lc}
\hline \hline & \\
ID & {$[\mathrm{Fe} / \mathrm{H}]_{M}$} \\
\hline & \\
BS 121 & $-1.00 \pm 0.04$ \\
HW 47 & $-1.06 \pm 0.04$ \\
HW 84 & $-1.00 \pm 0.03$ \\
HW 86 & $-0.97 \pm 0.05$ \\
L 4 & $-1.12 \pm 0.04$ \\
L 5 & $-0.96 \pm 0.06$ \\
L 6 & $-0.93 \pm 0.03$ \\
L 7 & $-1.01 \pm 0.06$ \\
L 17 & $-0.89 \pm 0.06$ \\
L 19 & $-1.03 \pm 0.05$ \\
L 27 & $-0.81 \pm 0.04$ \\
L 106 & $-0.77 \pm 0.13$ \\
L 108 & $-1.18 \pm 0.09$ \\
L 110 & $-1.10 \pm 0.07$ \\
L 111 & $-1.04 \pm 0.09$ \\
\hline \hline
\end{tabular}




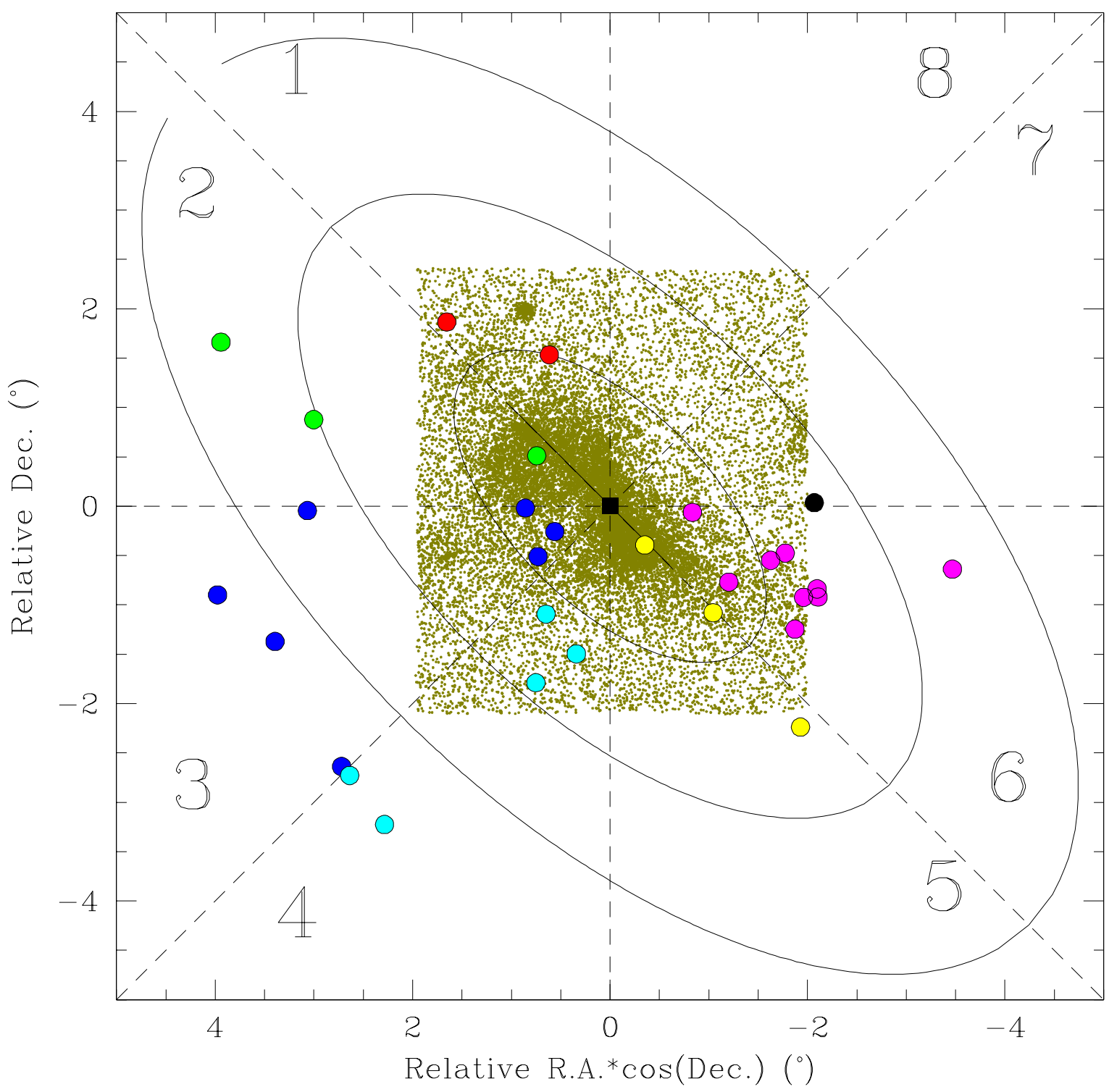

Fig. 1.- Relative position of our field sample (this work + P10). For reasons related to the metallicity gradient analysis (Section 5), we divide the figure in eight sectors and plotted with a different color fields located in each sector. 

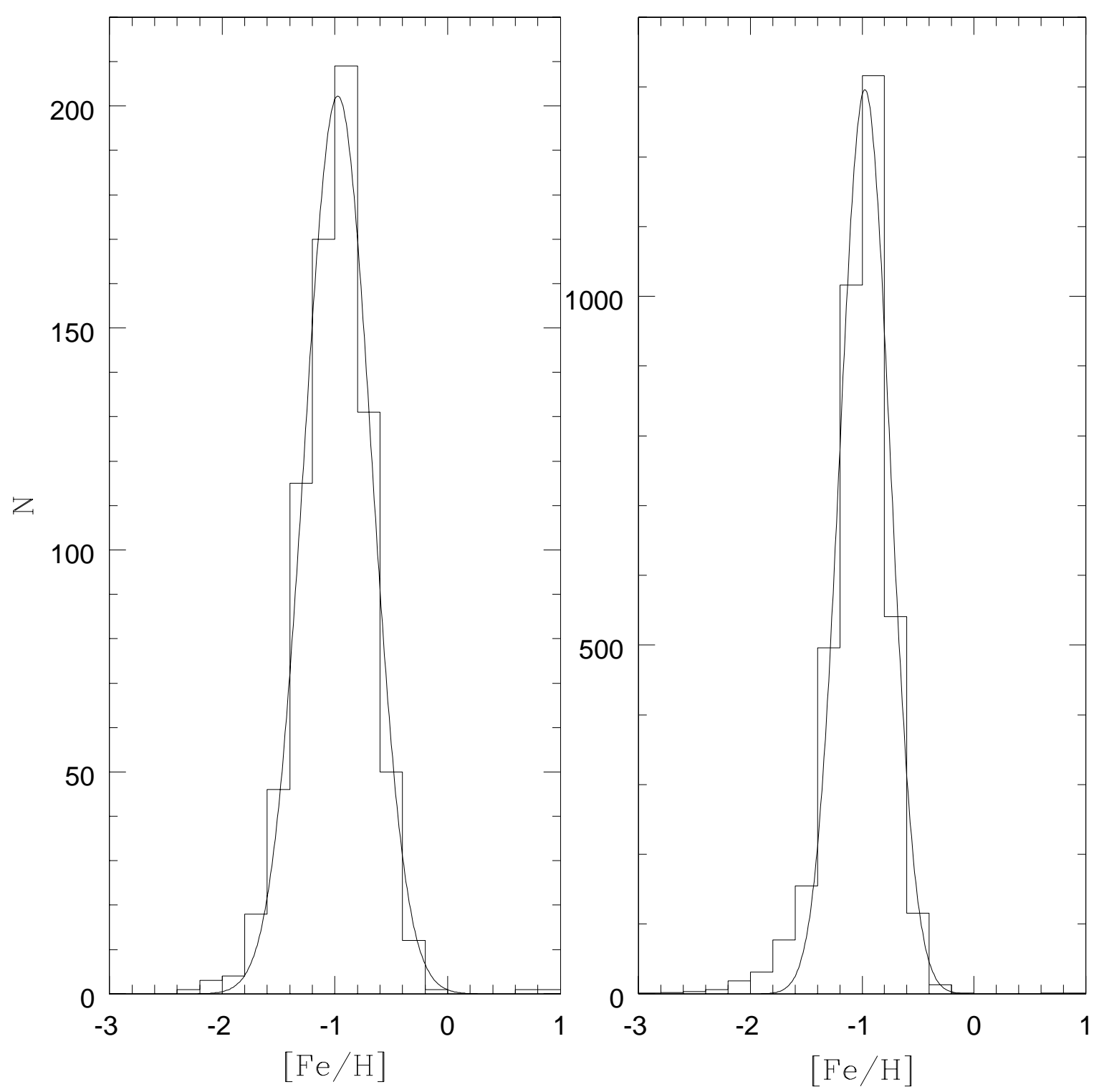

Fig. 2.- Metallicity distribution of all $750 \mathrm{SMC}$ field red giants studied in the present work and P10 (Left panel) and of our field giants plus 3037 giants studied by D14b (Right panel). In both figures the solid curve is the best Gaussian fit, which is generally a very good representation of the MD except for the most metal-poor stars, where there is a hint of an excess of stars in the observed MD. 


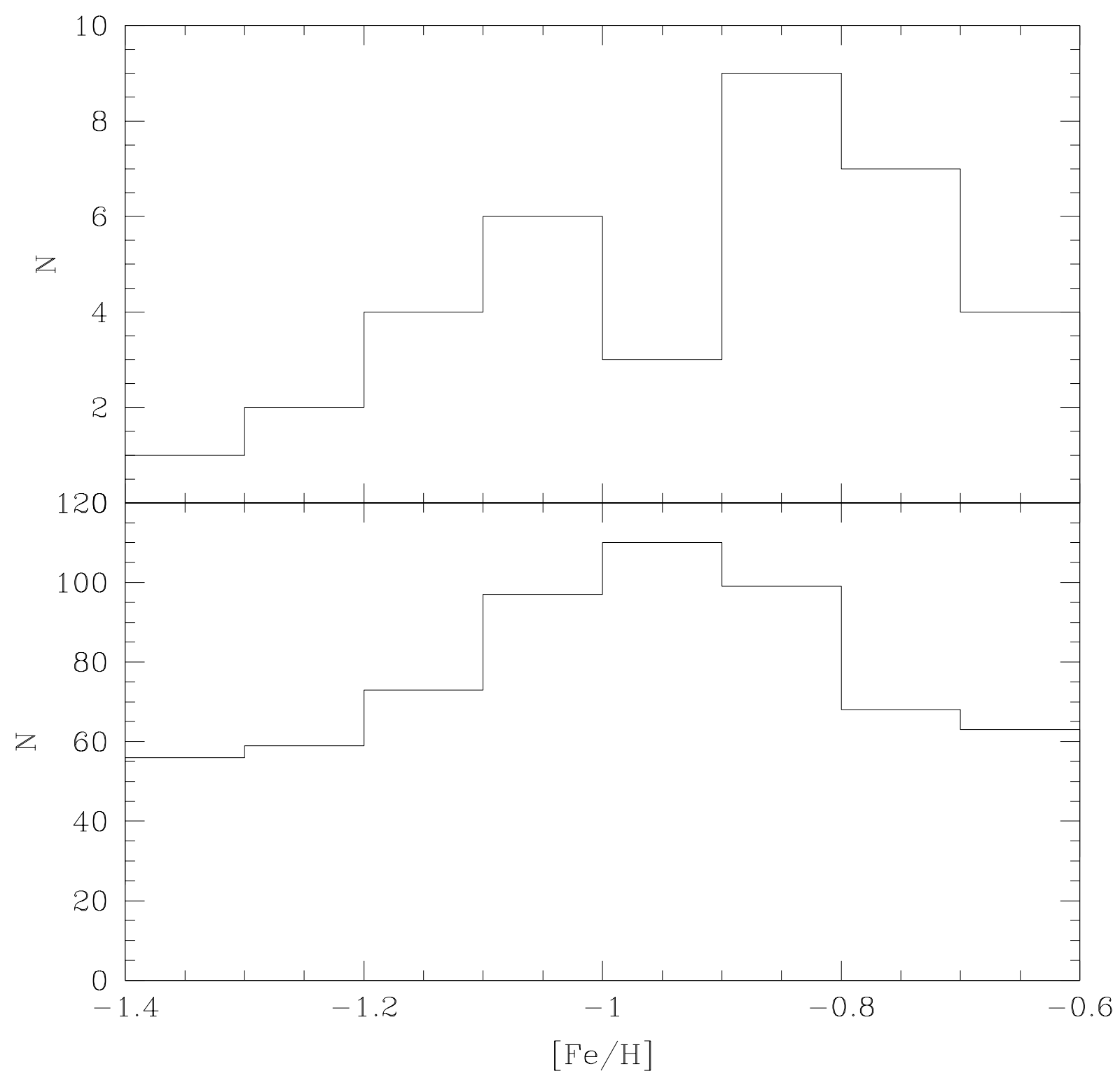

Fig. 3.- Metallicity distribution of our field giants (bottom) compared to the cluster metallicity distribution from P15 (top). 

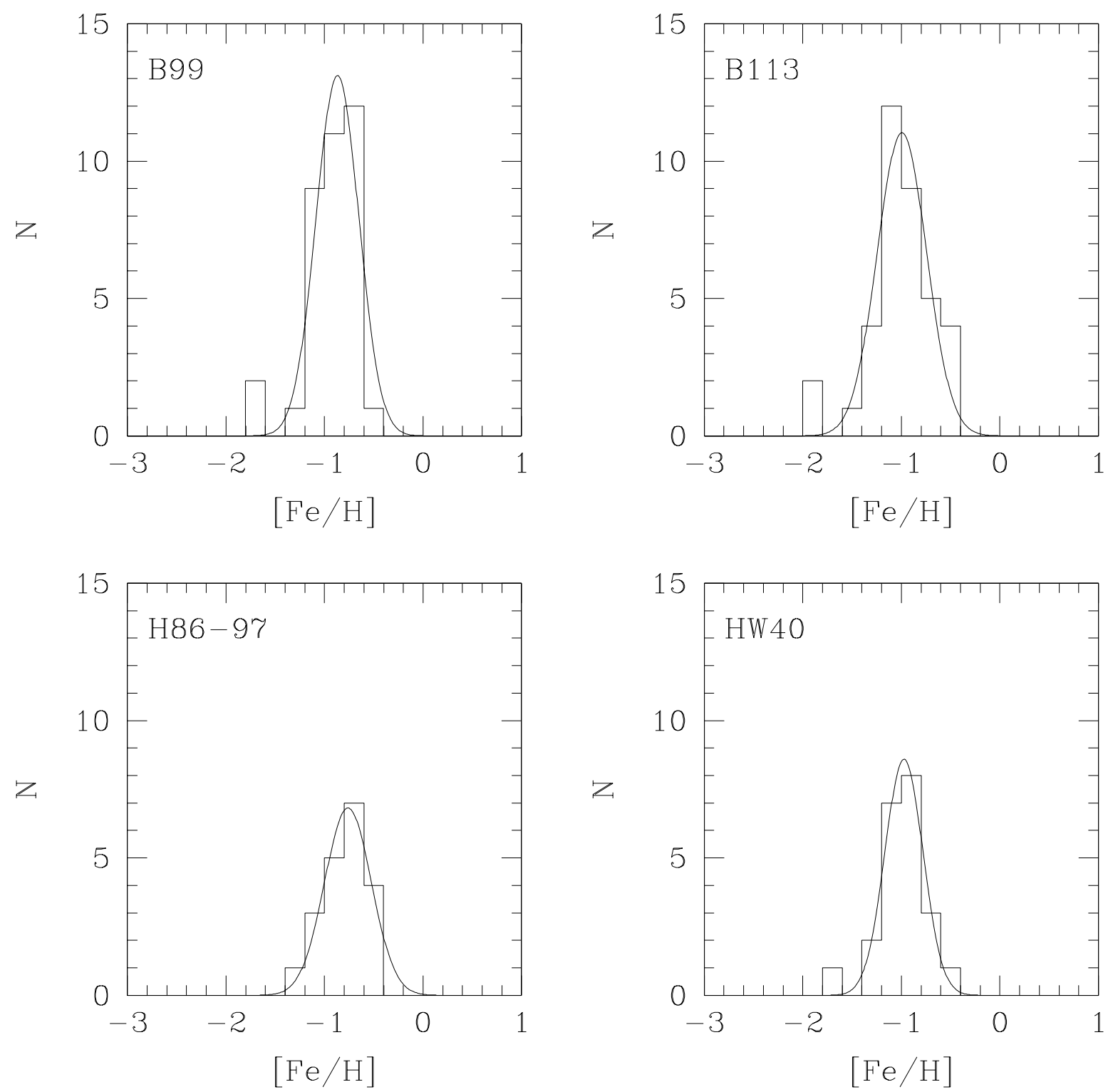

Fig. 4.- Metallicity distributions of the field stars surrounding clusters B99, B113, H86-97 and HW40. The corresponding Gaussian fits are shown in solid lines. 

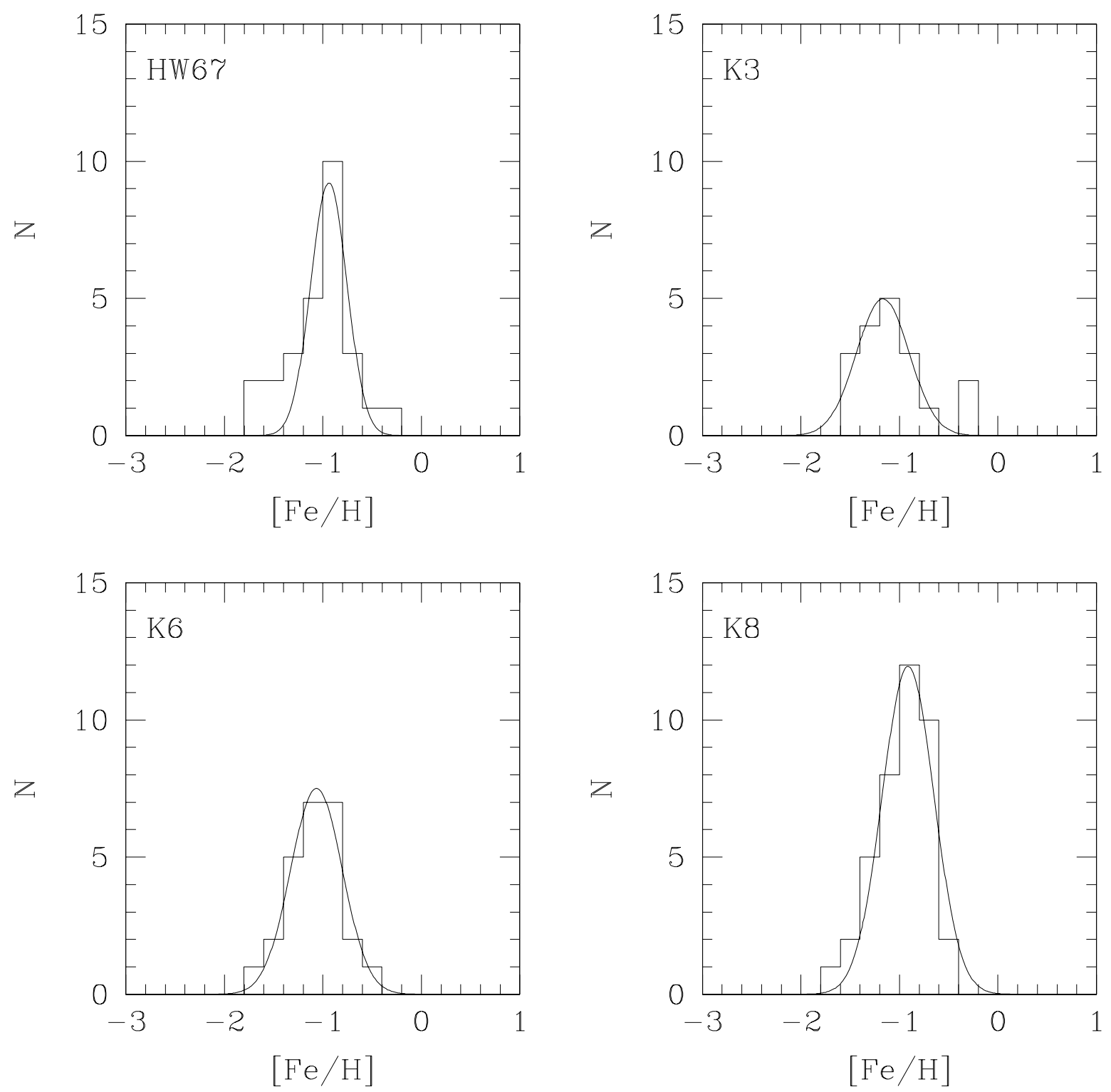

Fig. 5.- Same as Figure 4 but for stars surrounding clusters HW67, K3, K6 and K8. 

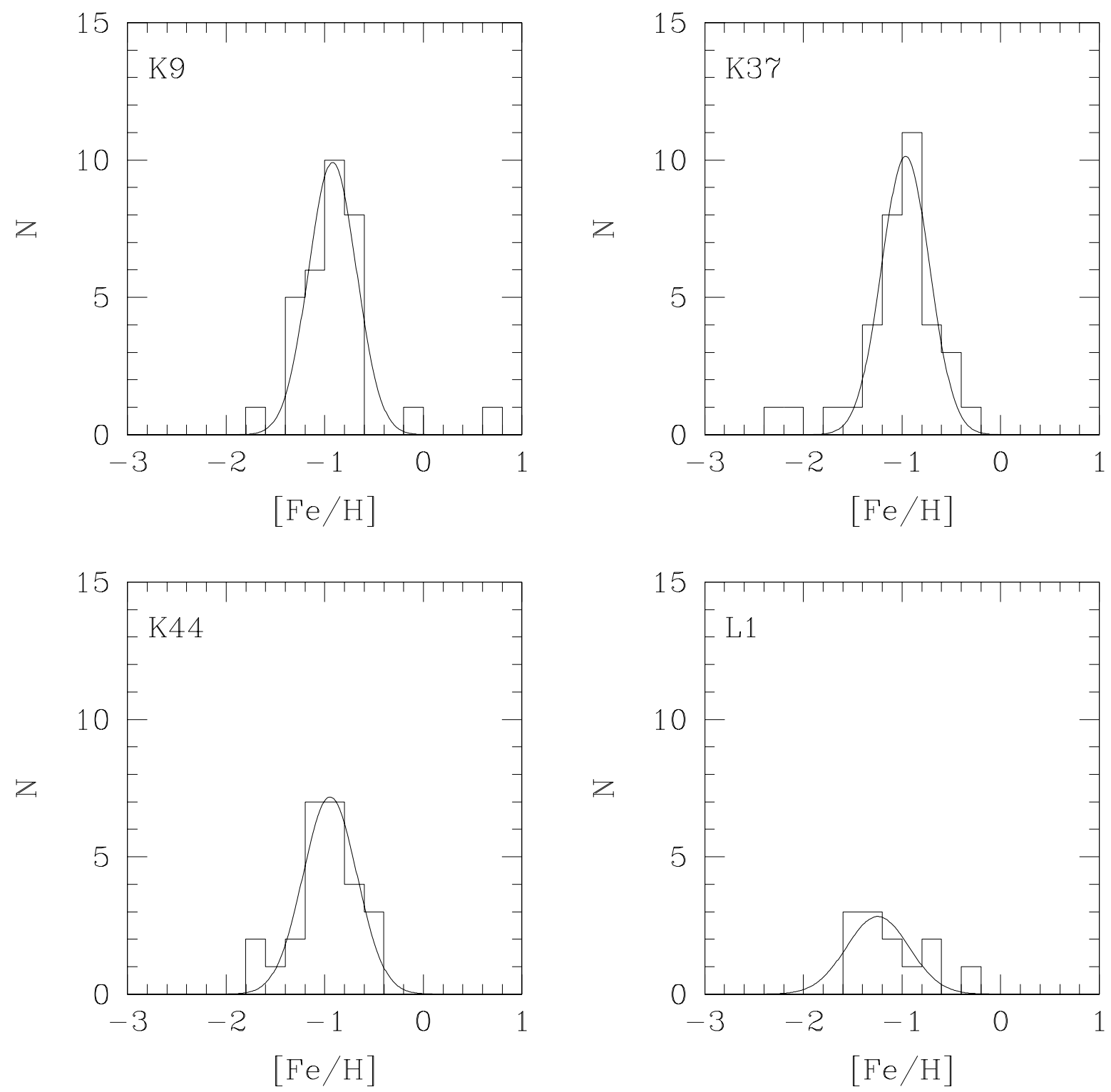

Fig. 6. - Same as Figure 4 but for stars surrounding clusters K9, K37, K44 and L1. 

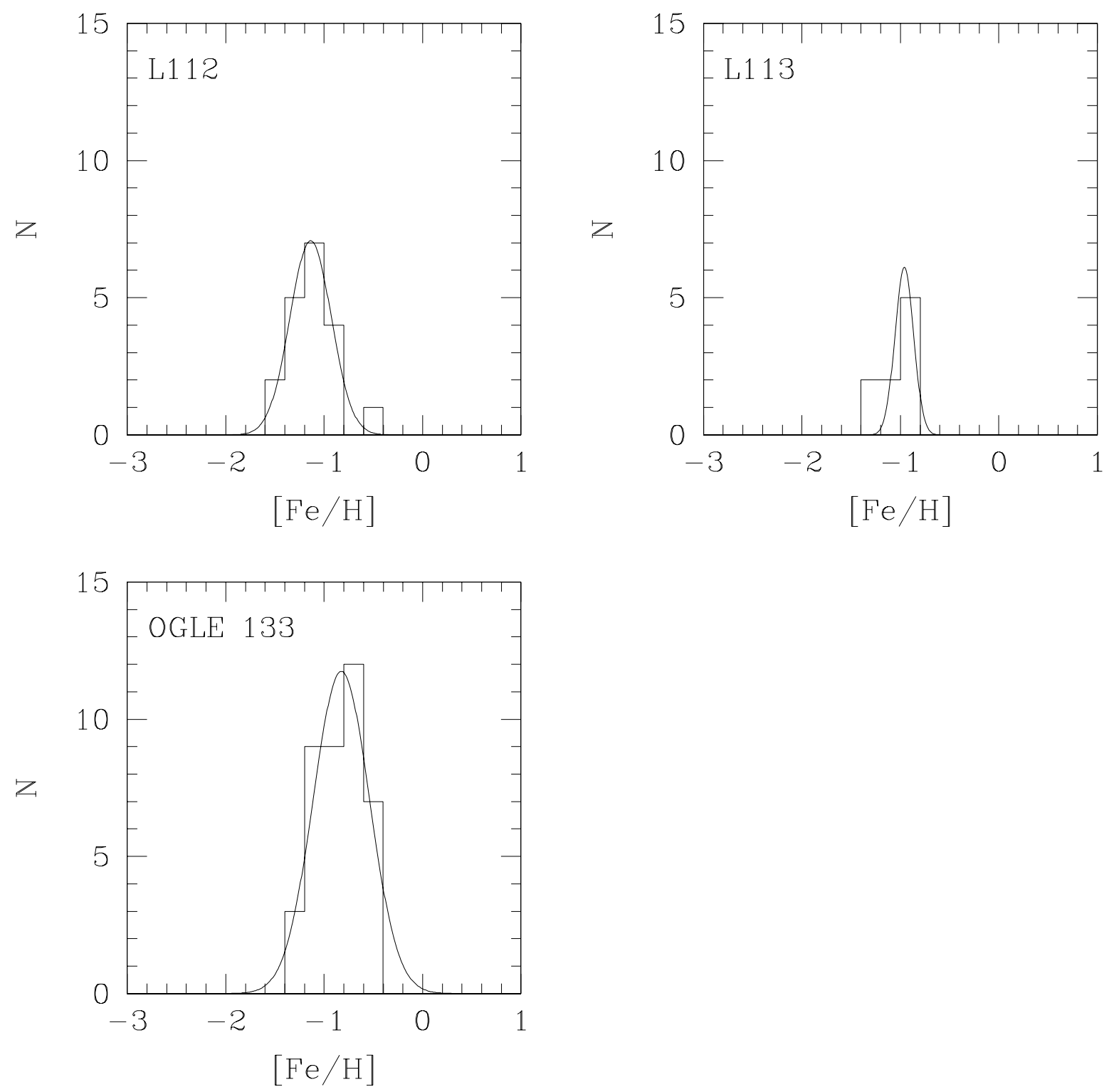

Fig. 7.- Same as Figure 4 but for stars surrounding clusters L112, L113 and OGLE 133. 


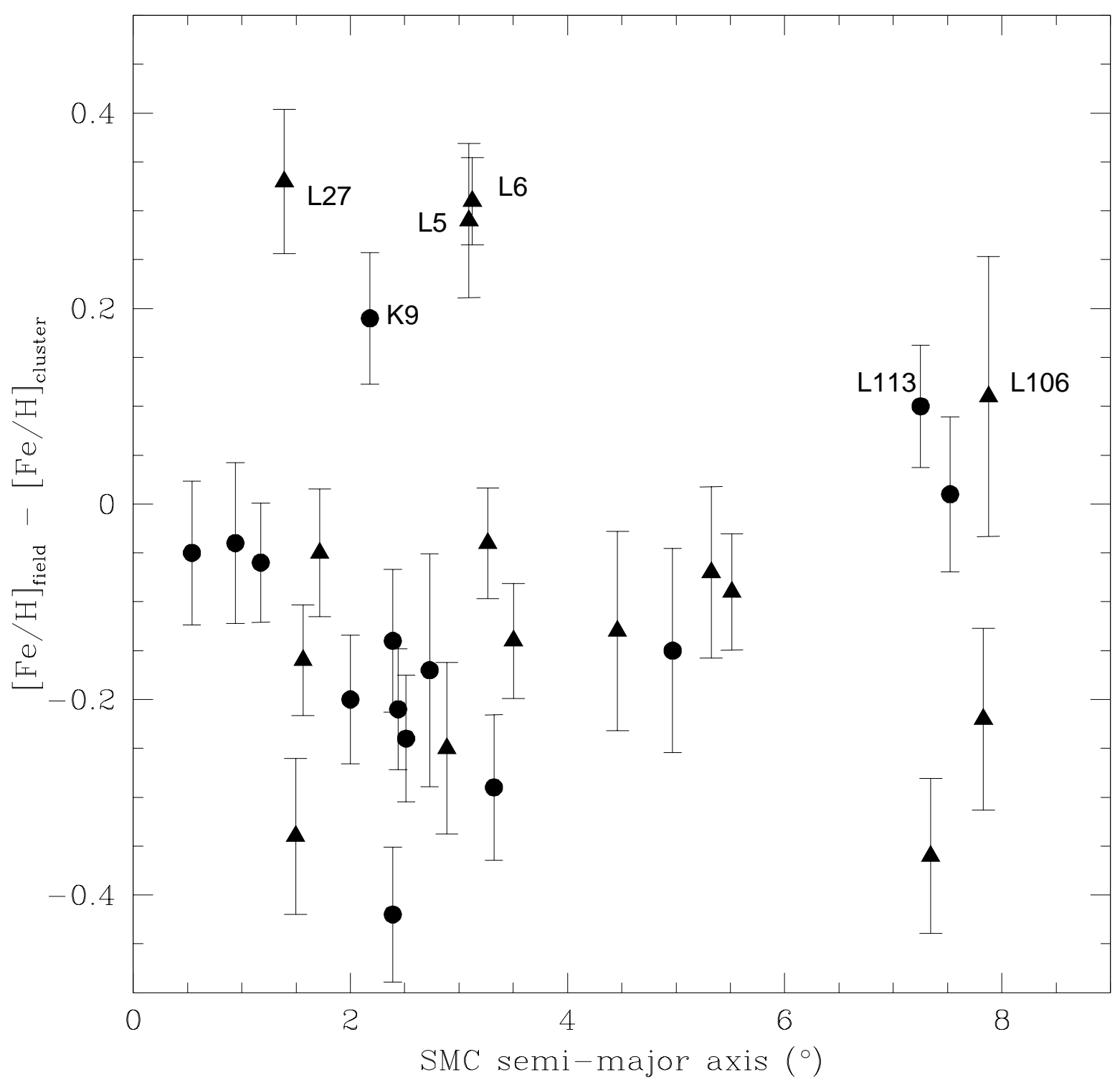

Fig. 8.- Difference between field and cluster metallicities as a function of the semi-major axis a. Triangles and circles denote star fields studied in P10 and in the present work, respectively. 


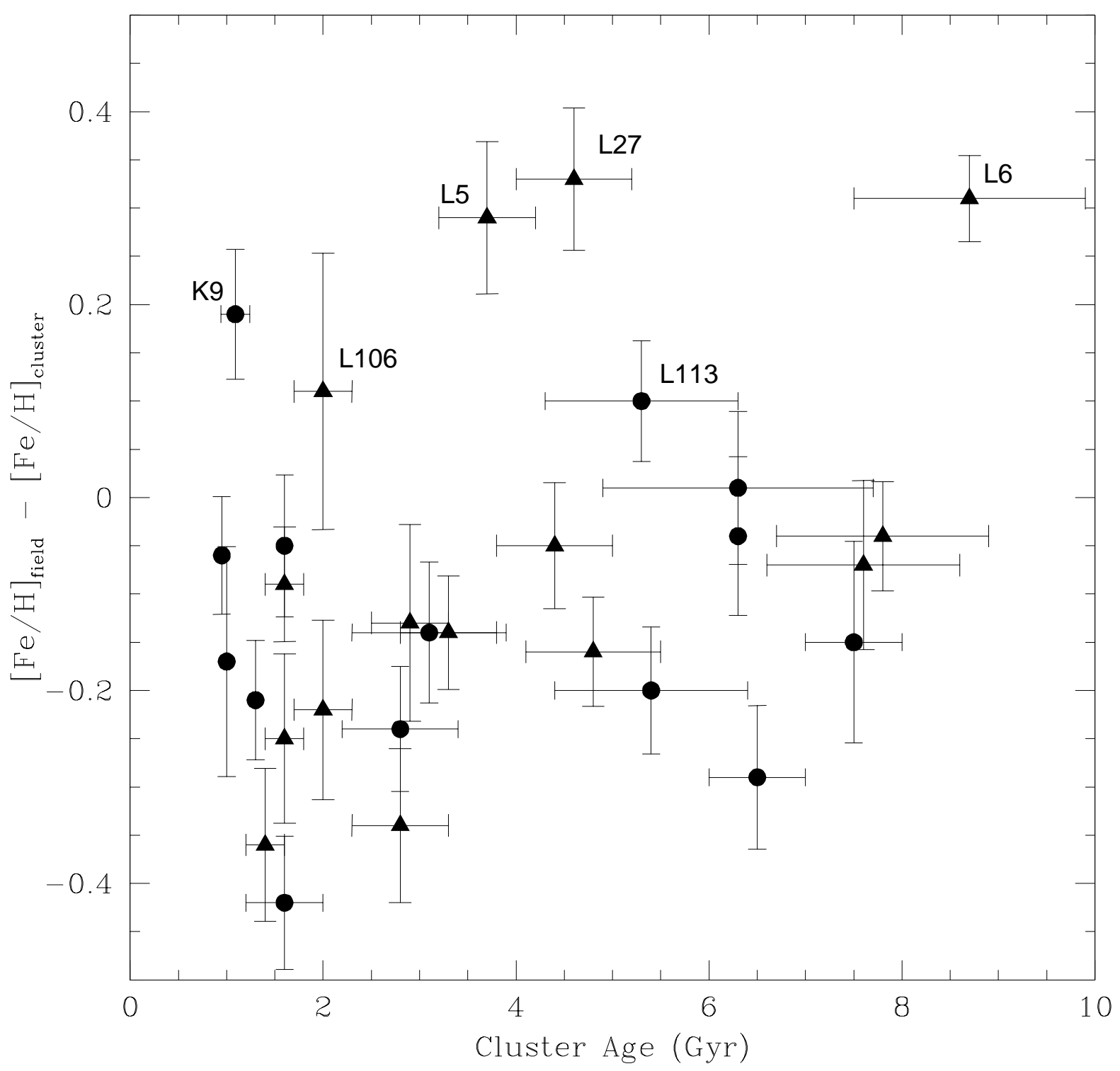

Fig. 9.- Difference between field and cluster metallicities as a function of cluster ages. Symbols are the same as Figure 8. 


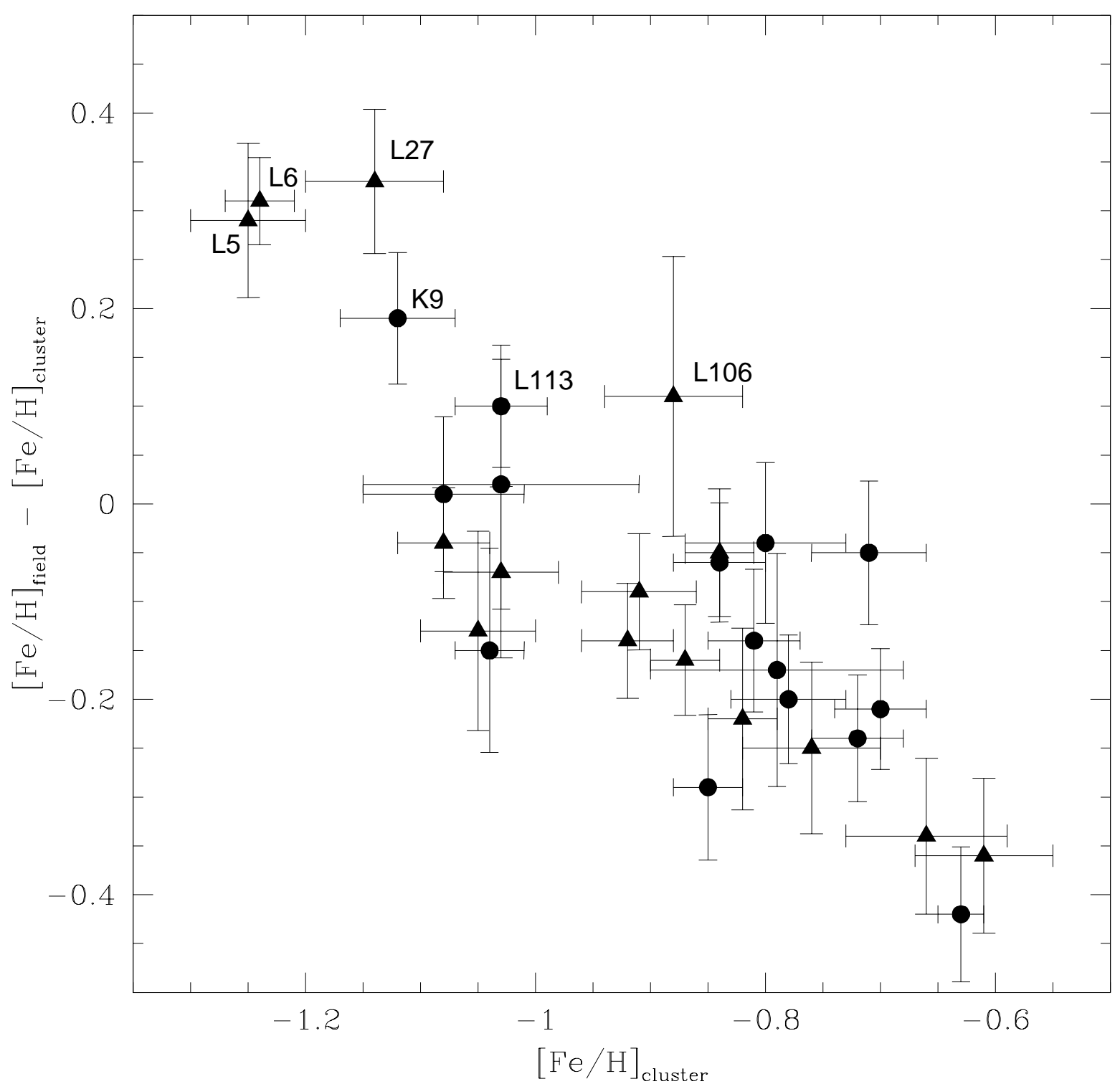

Fig. 10.- Difference between field and cluster metallicities as a function of cluster metallicities. Symbols are the same as Figure 8 

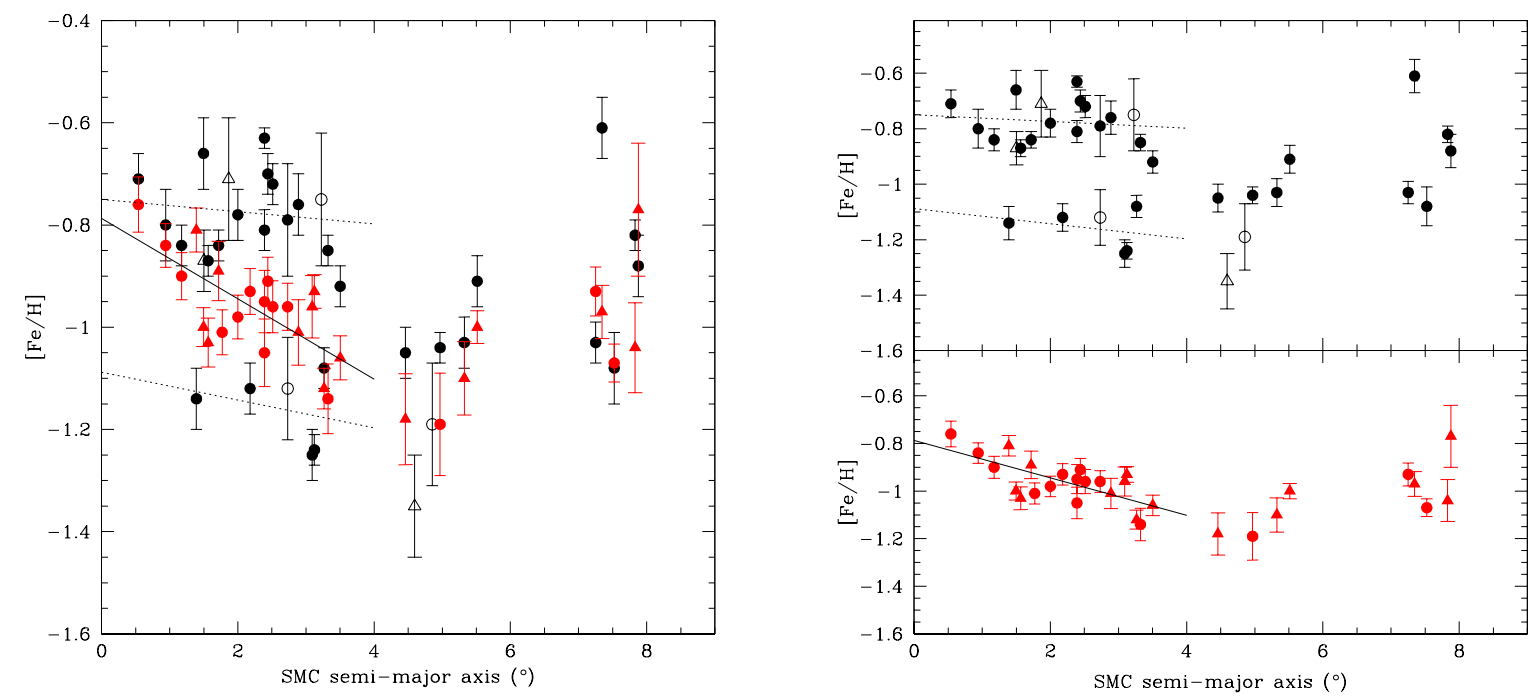

Fig. 11. - Left panel. Metallicities as a function of the semi-major axis $a$. Black and red symbols represent cluster and surrounding field metallicities, respectively. Cluster sample: filled circles represent clusters studied in P09 and P15, open circles and triangles those from Da Costa \& Hatzidimitriou (1998) and Glatt et al. (2008a,b), respectively. The field sample symbols are the same as in Figure 8. Field median metallicities are plotted. The solid line corresponds to the best linear fit performed considering only field stars, while dotted lines are the linear fit considering separately the two groups of clusters (metal-rich and metal-poor groups). Right panel. Same as the left panel. The two stellar populations (clusters above and fields below) are separated for a better visualization of the two dataset. 


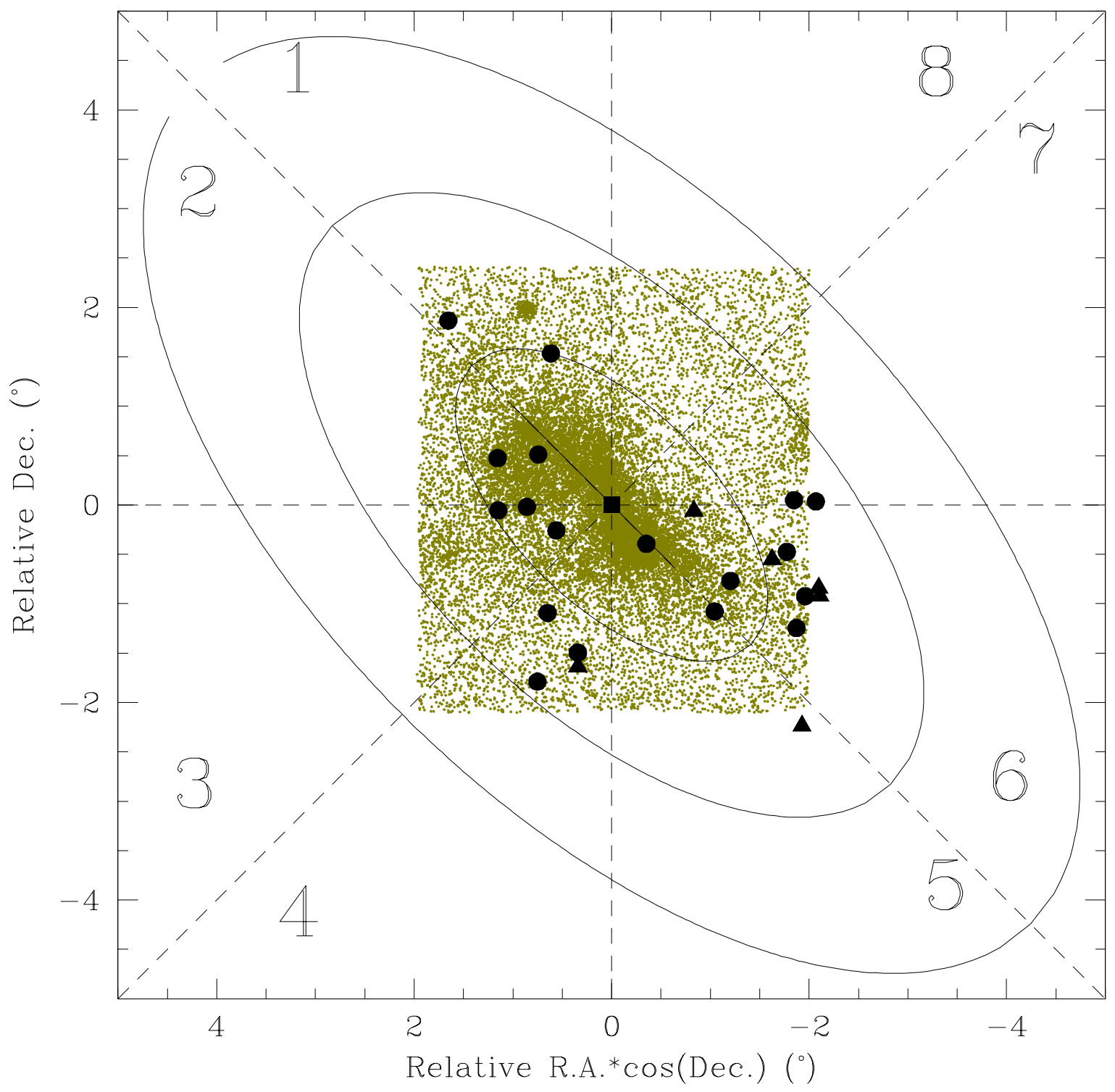

Fig. 12.- Distribution of clusters located in the inner region $\left(a<4^{\circ}\right)$. Triangles and circles represent clusters of the metal-poor and the metal-rich groups, respectively. 


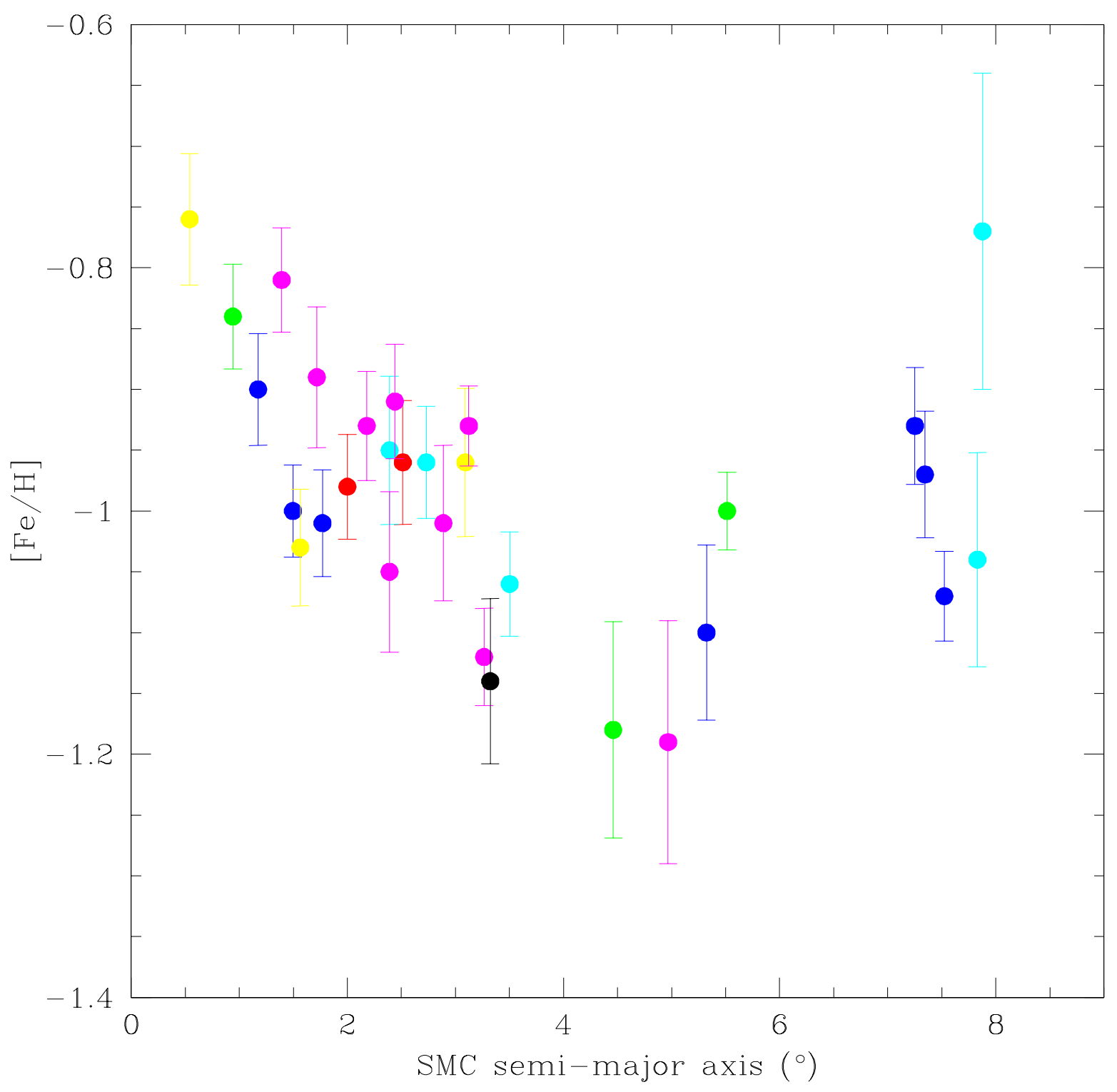

Fig. 13. - Metallicities as a function of the semi-major axis $a$ for field stars. Fields located in different positions of the SMC have been plotted in different colours (see Figure 1). 